\title{
Pseudospin symmetry and the relativistic harmonic oscillator
}

\author{
R. Lisboa and M. Malheiro \\ Instituto de Física, Universidade Federal Fluminense, 24210-340 Niterói, Brazil \\ A. S. de Castro \\ Departamento de Física e Química, Universidade Estadual Paulista, 12516-410 Guaratinguetá, São Paulo, Brazil \\ P. Alberto and M. Fiolhais \\ Departamento de Física and Centro de Física Computacional, Universidade de Coimbra, P-3004-516 Coimbra, Portugal
}

(Received 27 October 2003; published 27 February 2004)

\begin{abstract}
A generalized relativistic harmonic oscillator for spin 1/2 particles is studied. The Dirac Hamiltonian contains a scalar $S$ and a vector $V$ quadratic potentials in the radial coordinate, as well as a tensor potential $U$ linear in $r$. Setting either or both combinations $\Sigma=S+V$ and $\Delta=V-S$ to zero, analytical solutions for bound states of the corresponding Dirac equations are found. The eigenenergies and wave functions are presented and particular cases are discussed, devoting a special attention to the nonrelativistic limit and the case $\Sigma=0$, for which pseudospin symmetry is exact. We also show that the case $U=\Delta=0$ is the most natural generalization of the nonrelativistic harmonic oscillator. The radial node structure of the Dirac spinor is studied for several combinations of harmonic-oscillator potentials, and that study allows us to explain why nuclear intruder levels cannot be described in the framework of the relativistic harmonic oscillator in the pseudospin limit.
\end{abstract}

DOI: 10.1103/PhysRevC.69.024319

PACS number(s): 21.10.Hw, 21.60.Cs, 03.65.Pm

\section{INTRODUCTION}

The harmonic-oscillator potential for relativistic spin $1 / 2$ particles has received considerable attention by many groups. The subject is of broad interest since the relativistic harmonic oscillator plays a role in several areas, namely, in nuclear and particle physics. In particular, it is the central potential of the nuclear shell model, and it has also been used as the binding (and confining) two-body potential for quarks, with applications in meson and baryon spectroscopy.

A special type of harmonic-oscillator potential is achieved by replacing the linear momentum operator $\boldsymbol{p}$ in the Dirac equation by $\boldsymbol{p}-i \beta m \omega \boldsymbol{r}$, where $\beta$ is the usual Dirac matrix. This replacement results in a second order differential equation for the upper and lower components of the Dirac spinor containing a quadratic potential in the radial coordinate $r$. Besides the quadratic potential, that second order differential equation contains a constant spin-orbit term, meaning that, in the nonrelativistic limit, in which only the upper component survives, the degeneracy of the energy levels is different from the one found in the nonrelativistic harmonic oscillator. As explained in the following section, that potential may arise from a Lorentz tensor interaction in spinor space. In Ref. [1] we give a list of references where this kind of potential was initially studied. Moshinsky and Szczepaniak [2] christened it Dirac oscillator and renewed to a great extent the interest in the topic. The Dirac oscillator has been applied to quark confinement and supersymmetry [3] and hadron spectroscopy [4]. Its group symmetries have been studied in Ref. [5], its solutions in $2+1$ dimensions and coupling to magnetic field were worked out in Ref. [6], and finally its wave packets and thermodynamical properties in $1+1$ dimensions were studied in Ref. [7].

Another possibility to introduce a harmonic potential in the Dirac equation is by mixing vector and scalar harmonic potentials with equal magnitude and sign, aiming at obtaining a quadratic potential in the Schrödinger-like second order differential equation for each spinor component [8]. Kukulin et al. [9] generalized the problem by considering a vectorscalar harmonic potential plus the Dirac oscillator. More recently, there has been a wide interest in relativistic potentials involving mixtures of vector and scalar potentials with opposite signs. The interest lies on attempts to explain the pseudospin symmetry in nuclear physics. Cheng et al. [10], using a Dirac Hamiltonian with scalar $S$ and vector $V$ potentials quadratic in space coordinates, found a harmonic-oscillatorlike second order equation which can be solved analytically for $\Delta=V-S=0$, as considered before by Kukulin [9], and also for $\Sigma=S+V=0$. Very recently, Ginocchio solved the triaxial, axial, and spherical harmonic oscillators for the case $\Delta=0$ and applied it to the study of antinucleons embedded in nuclei [11]. The case $\Sigma=0$ is particularly relevant in nuclear physics, since it is usually pointed out as a necessary condition for occurrence of pseudospin symmetry in nuclei $[12,13]$.

In this work we shall consider a Dirac Hamiltonian that generalizes the previous ones by simultaneously introducing radial quadratic potentials for $\Delta, \Sigma$ and a linear radial potential for $U$, the tensor potential defined in the following section. We will study the cases with $\Delta=0$ and $\Sigma=0$, for which analytical bound solutions do exist, including also, as particular cases, the Kukulin potential and the original Dirac oscillator. The eigenenergies and eigenfunctions are obtained analytically in the general case. Next we shall analyze particular cases and pay a special attention to the nonrelativistic limits and to the case $U=\Sigma=0$, for which pseudospin symmetry is exact and there are still bound states, as opposed to what happens with nuclear mean fields. From our analysis we also show that the case $U=\Delta=0$ is a more natural way to introduce a harmonic oscillator in the Dirac equation than the 
usual way with $U \neq 0$ and $\Sigma=\Delta=0$. The eigenenergies have the usual degeneracy of the nonrelativistic case and the upper component a form similar to the nonrelativistic wave function. Actually, by letting the harmonic-oscillator frequency to become small compared with the mass, we obtain exactly the eigenvalues and wave functions of the nonrelativistic harmonic oscillator.

We also present the node structure of the radial wave functions, motivated by a study carried on in Ref. [14] for $V$ and $S$ radial potentials vanishing as $r \rightarrow \infty$. In order to find reasons for the unusual radial node structures in some particular cases, we obtain the relations between the radial nodes of the upper and lower components of the Dirac spinors by inspection of their analytical forms, and illustrate those relations by plots of several of those components. We draw conclusions regarding the impossibility to describe the so-called intruder states by harmonic-oscillator potentials with exact pseudospin symmetry.

This paper is organized as follows. In Sec. II we present the general Dirac equation with scalar and vector potentials with harmonic-oscillator form, proportional to $r^{2}$, and a tensor potential, linear in $r$. We then obtain and discuss the solutions of this equation for $\Delta=0$ and $\Sigma=0$, respectively. The Dirac oscillator is presented in Sec. III A. In Sec. III B and III C we look into the particular cases $\Delta=0$ and $\Sigma=0$ in the absence of the tensor potential. We analyze the nonrelativistic limits, showing that such a limit does not exist in the case $\Sigma=0$, up to first order in the oscillator frequency divided by the mass. This result is connected to the pseudospin symmetry, as discussed in more detail in Sec. IV. In the same section we also present an analysis of the intruder states and show the impossibility to describe them within the harmonic oscillator in the case of exact pseudospin symmetry. Finally, our conclusions are summarized in Sec. V.

\section{GENERALIZED RELATIVISTIC HARMONIC OSCILLATOR}

The time-independent Dirac equation for a spin $1 / 2$ fermion with energy $\mathcal{E}$, in the presence of a potential, reads

$$
H_{D} \Psi=\mathcal{E} \Psi,
$$

where the Dirac Hamiltonian is

$$
H_{D}=\boldsymbol{\alpha} \cdot \boldsymbol{p}+\beta m+\mathcal{V} .
$$

In this Hamiltonian, $m$ is the fermion mass, $\boldsymbol{p}$ is the momentum operator, and $\boldsymbol{\alpha}$ and $\beta$ are $4 \times 4$ matrices which, in the usual representation, take the form

$$
\boldsymbol{\alpha}=\left(\begin{array}{cc}
0 & \boldsymbol{\sigma} \\
\boldsymbol{\sigma} & 0
\end{array}\right), \quad \beta=\left(\begin{array}{cc}
I & 0 \\
0 & -I
\end{array}\right) .
$$

Here $\boldsymbol{\sigma}$ is a three-vector whose components are the Pauli matrices, and $I$ stands for the $2 \times 2$ identity matrix. The matrix potential $\mathcal{V}$ in Eq. (2) may, in general, be written as a linear combination of sixteen linearly independent matrices, classified according to their properties under Lorentz transformations: scalar, pseudoscalar, vector, pseudovector, and tensor. In the following, we exclude the pseudoscalar and pseudovector potentials, only considering spherically symmetric potentials, for which the total angular momentum of the system is constant. In spite of being called spherically symmetric potentials, they do not necessarily depend only on the radial coordinate $r=|\boldsymbol{r}|$, because of their matrix structure. Indeed, the following potential:

$$
\mathcal{V}(\boldsymbol{r})=V(r)+\beta S(r)+i \beta \boldsymbol{\alpha} \cdot \hat{\boldsymbol{r}} U(r)
$$

is spherically symmetric in this sense, because it commutes with the total angular momentum $\boldsymbol{J}=\boldsymbol{L}+\boldsymbol{S}$, where $\boldsymbol{L}$ and $\boldsymbol{S}$ are the orbital and spin angular momentum operators, respectively. In the last term, $\hat{\boldsymbol{r}}=\boldsymbol{r} / r$, and the radial functions in Eq. (4) are named after the properties their respective terms have under Lorentz transformations: $V$ corresponds to the time component of a vector potential, $S$ is a scalar potential, and $U$ is a tensor potential. It is important to point out that the Dirac equation for the potential in Eq. (4) is invariant under spatial inversion, hence the eigenstates have definite parity.

It is worth showing where the tensor character of the last term in Eq. (4) comes from. The interaction Lagrangian for the tensor coupling of a Dirac spinor $\Psi$ with an external field is

$$
\frac{f}{4 M} \bar{\Psi} \sigma^{\mu \nu} \Psi \phi_{\mu \nu},
$$

where $\phi_{\mu \nu}=\partial_{\mu} \phi_{\nu}-\partial_{\nu} \phi_{\mu}, \sigma^{\mu \nu}=(i / 2)\left[\gamma^{\mu}, \gamma^{\nu}\right]$, and $\gamma^{\nu}$ are the Dirac matrices. Considering only the time component of the vector potential $\phi^{\mu}, \phi^{0}$, the corresponding term leads to the following potential in the single-particle (Dirac) Hamiltonian:

$$
i \frac{f}{2 M} \beta \boldsymbol{\alpha} \cdot \nabla \phi^{0} .
$$

For a static radial potential $\phi^{0}$, the tensor potential $U(r)$ in Eq. (4) is $U(r)=f /(2 M)\left(d \phi^{0} / d r\right)$. This term can be used to describe a particle with an anomalous magnetic moment. Indeed, the anomalous magnetic interaction has the form $-i \mu \beta \boldsymbol{\alpha} \cdot \nabla \varphi(r)$, where $\mu$ is the anomalous magnetic moment, in units of Bohr magnetons, and $\varphi$ is the electric potential, i.e., the time component of the electromagnetic vector potential, $A^{\mu}$. Therefore, the interaction $-i m \omega \beta \boldsymbol{\alpha} \cdot \boldsymbol{r}$ (notice that $\beta$ and $\boldsymbol{\alpha}$ are now written in reverse order), which defines the "Dirac oscillator" mentioned above, could be conceived as an anomalous magnetic interaction generated by an electrostatic harmonic potential.

In summary, for this type of potentials, the Hamiltonian, the parity operator, and the total angular momentum form a complete set of commuting observables. Accordingly, the eigenstates can be classified by the parity $( \pm)$, by the total angular momentum $j$, and its third component $m$, quantum numbers. Under these circumstances it is natural to use spherical coordinates and the spinor, which is the solution of the Dirac equation (1), can be generally written as 


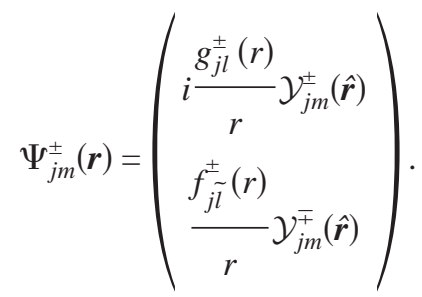

In this expression, $\mathcal{Y}_{j m}$ are the so-called spinor spherical harmonics. They result from the coupling of the twodimensional spinors to the eigenstates of orbital angular momentum and form a complete orthonormal set. The orbital angular momentum quantum numbers $l$ and $\tilde{l}$ refer to the upper and lower components, respectively. The normalization of the Dirac spinor in Eq. (7) implies the following normalization condition on the upper and the lower radial functions:

$$
\int_{0}^{\infty}\left(\left|g_{j}^{ \pm}\right|^{2}+\left|f_{j l}^{ \pm}\right|^{2}\right) d r=1
$$

so that $g_{j l}^{ \pm}$and $f_{j l}^{ \pm}$should be square-integrable functions.

Using the operator $\boldsymbol{\alpha} \cdot \boldsymbol{p}$ in the spherical-polar form, Eq. (1) can be brought to the form

$$
\begin{gathered}
\left\{-i \boldsymbol{\alpha} \cdot \hat{\boldsymbol{r}}\left[\frac{\partial}{\partial r}+\frac{1}{r}(1+\beta K)\right]+\beta[m+S(r)]+V(r)\right. \\
+i \beta \boldsymbol{\alpha} \cdot \hat{\boldsymbol{r}} U(r)\} \Psi=\mathcal{E} \Psi,
\end{gathered}
$$

where we have introduced the operator $K=-\beta(\Sigma \cdot L+1)$ whose eigenvalues are $\kappa= \pm(j+1 / 2)$. Here $\Sigma$ refers to the $4 \times 4$ matrix whose block diagonal elements are the Pauli matrices, $\boldsymbol{\sigma}$, and whose off-diagonal blocks are zero. More explicitly, the spin-orbit coupling quantum number $\kappa$ is related to the orbital angular momentum quantum number by

$$
\kappa=\left\{\begin{array}{rr}
-(l+1)=-(j+1 / 2), & j=l+1 / 2 \quad(\kappa<0) \\
\text { aligned spin } \\
l=+(j+1 / 2), & j=l-1 / 2(\kappa>0) \\
\text { unaligned spin } .
\end{array}\right.
$$

Actually, the quantum number $\kappa$ completely determines $j, l$, $\tilde{l}$ and, thus, the parity $(-1)^{l}$ :

$$
\begin{gathered}
j=|\kappa|-\frac{1}{2}, \\
l=|\kappa|+\frac{1}{2}\left(\frac{\kappa}{|\kappa|}-1\right), \\
\tilde{l}=l-\frac{\kappa}{|\kappa|} .
\end{gathered}
$$

Notice that $\tilde{l}$ is given by the same formula as $l$, but with $-\kappa$ instead of $\kappa$. Accordingly, the Dirac spinors in Eq. (7) can be labeled just with $\kappa$ and $m$, i.e.,

$$
\Psi_{\kappa m}(\boldsymbol{r})=\left(\frac{i \frac{g_{\kappa}(r)}{r} \mathcal{Y}_{\kappa m}(\hat{\boldsymbol{r}})}{\frac{f_{\kappa}(r)}{r} \mathcal{Y}_{-\kappa m}(\hat{\boldsymbol{r}})}\right) .
$$

Using the property $\boldsymbol{\sigma} \cdot \hat{\boldsymbol{r}} \mathcal{Y}_{\kappa m}=-\mathcal{Y}_{-\kappa m}$, the Dirac equation in Eq. (1) may be reduced to a set of two coupled first order ordinary differential equations for the radial upper and lower components $g_{\kappa}$ and $f_{\kappa}$, namely,

$$
\begin{gathered}
{\left[\frac{d}{d r}+\frac{\kappa}{r}+U(r)\right] g_{\kappa}(r)=[\mathcal{E}+m-\Delta(r)] f_{\kappa}(r),} \\
{\left[\frac{d}{d r}-\frac{\kappa}{r}-U(r)\right] f_{\kappa}(r)=-[\mathcal{E}-m-\Sigma(r)] g_{\kappa}(r),}
\end{gathered}
$$

where we have introduced the "sum" and the "difference" potentials defined by

$$
\Sigma=V+S \text { and } \Delta=V-S .
$$

Using the expression for $f_{\kappa}$ obtained from Eq. (13) and inserting it in Eq. (14) we arrive at the following second order differential equation for $g_{\kappa}$ :

$$
\begin{aligned}
\left\{\frac{d^{2}}{d r^{2}}\right. & -\frac{\kappa(\kappa+1)}{r^{2}}+\frac{\Delta^{\prime}}{\mathcal{E}+m-\Delta(r)}\left[\frac{d}{d r}+\frac{\kappa}{r}+U(r)\right] \\
& \left.-2 \kappa \frac{U(r)}{r}+U^{\prime}(r)-U^{2}(r)\right\} g_{\kappa}(r) \\
= & -[\mathcal{E}-m-\Sigma(r)][\mathcal{E}+m-\Delta(r)] g_{\kappa}(r),
\end{aligned}
$$

where the prime means derivative with respect to $r$. In a similar fashion, i.e., using again both Eqs. (13) and (14), a second order differential equation for the lower component is obtained:

$$
\begin{aligned}
\left\{\frac{d^{2}}{d r^{2}}\right. & -\frac{\kappa(\kappa-1)}{r^{2}}+\frac{\Sigma^{\prime}}{\mathcal{E}-m-\Sigma(r)}\left[\frac{d}{d r}-\frac{\kappa}{r}-U(r)\right] \\
& \left.-2 \kappa \frac{U(r)}{r}-U^{\prime}(r)-U^{2}(r)\right\} f_{\kappa}(r) \\
= & -[\mathcal{E}-m-\Sigma(r)][\mathcal{E}+m-\Delta(r)] f_{\kappa}(r) .
\end{aligned}
$$

In this paper we shall consider harmonic-oscillator potentials, meaning that potentials $\Sigma$ and $\Delta$ are quadratic in $r$ and the potential $U$ linear in $r$. With all these potentials in place, Eqs. (16) and (17) have to be solved numerically because of the quartic potentials in $r$ arising from the product $[\mathcal{E}-m$ $-\Sigma(r)][\mathcal{E}+m-\Delta(r)]$ and the terms with derivatives of $\Delta$ and $\Sigma$. However, either for $\Delta=0$ or for $\Sigma=0$ the solutions are analytical since, in the first case, there are only quadratic potentials in the second order equation for $g_{\kappa}$ [Eq. (16)], and, in the second case, the same happens in Eq. (17) for $f_{\kappa}$. The case $\Delta=U=0$ allows us to get, in the nonrelativistic limit, the spectrum and wave functions of the central nonrelativistic harmonic oscillator, i.e, Eq. (16) becomes the radial Schrödinger equation for the three-dimensional harmonic oscillator. For $U=0$, both cases $\Sigma=0$ and $\Delta=0$ correspond to 
SU(2) symmetries of the Dirac equation $[15,16]$. The case $\Sigma=0$ is related to the pseudospin symmetry in nuclei.

The aim of this paper is to study such analytical solutions and, in particular, to draw conclusions on the required conditions for the pseudospin symmetry to show up.

Let us first consider

$$
\Sigma(r)=\frac{1}{2} m \omega_{1}^{2} r^{2}, \quad \Delta(r)=0, \quad U(r)=m \omega_{2} r,
$$

where $\omega_{2}$ (a real number) is the frequency related to the tensor potential $U$ and $\omega_{1}$ (a non-negative number ${ }^{1}$ ) the frequency related to the "sum" potential $\Sigma$. Actually, this case was already considered by Kukulin et al. [9] for $\omega_{2}>0$. The possibility of Dirac bound states with a linear tensor potential, which can be unbounded from below, is due to the appearance of $U^{2}$ in Eqs. (16) and (17). It is noteworthy that the different signs of $\omega_{2}$ give rise to different possibilities of signs for the spin-orbit coupling. Equation (16) for the upper component takes the form

$$
\begin{aligned}
& \left\{\frac{d^{2}}{d r^{2}}-\frac{\kappa(\kappa+1)}{r^{2}}-\left[\frac{1}{2} m \omega_{1}^{2}(\mathcal{E}+m)+m^{2} \omega_{2}^{2}\right] r^{2}-(2 \kappa-1) m \omega_{2}\right. \\
& \left.+\left(\mathcal{E}^{2}-m^{2}\right)\right\} g_{\kappa}(r)=0 .
\end{aligned}
$$

It is convenient to introduce the following new variable and parameters:

$$
\begin{gathered}
y=\sqrt{\frac{m(\mathcal{E}+m)}{2}} \Omega r^{2}=a^{2} r^{2}, \\
\Omega=\sqrt{\frac{2 m}{\mathcal{E}+m} \omega_{2}^{2}+\omega_{1}^{2}}, \\
\lambda=\frac{(2 \kappa-1) m \omega_{2}-\left(\mathcal{E}^{2}-m^{2}\right)}{a^{2}},
\end{gathered}
$$

which allows us to write Eq. (19) in the simpler form

$$
\left\{4 y \frac{d^{2}}{d y^{2}}+2 \frac{d}{d y}-\frac{l(l+1)}{y}-y-\lambda\right\} g_{\kappa}(y)=0,
$$

since $\kappa(\kappa+1)=l(l+1)$. An asymptotic analysis suggests searching for solutions of the type

$$
g_{\kappa}(y)=A e^{-y / 2} y^{(l+1) / 2} \Gamma(y),
$$

where $\Gamma(y)$ is a function yet to be determined and $A$ a normalization constant determined by Eq. (8). Inserting this expression back into Eq. (23), the equation for $\Gamma(y)$ reads

\footnotetext{
${ }^{1}$ In fact, this condition can be relaxed to demand that $\omega_{1}$ be just a real number, since the results do not depend on the sign of $\omega_{1}$.
}

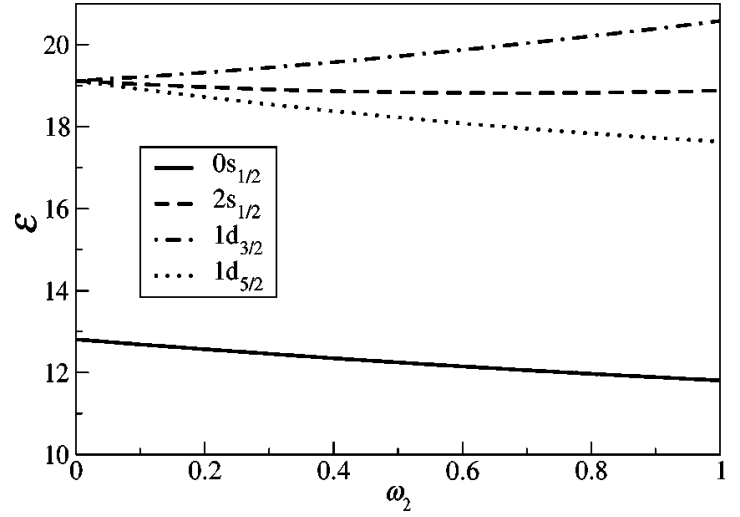

FIG. 1. Energy levels for $\Delta=0$ with $\omega_{1}=2$ and $m=10$ as a function of $\omega_{2}$.

$$
\left[y \frac{d^{2}}{d y^{2}}+\left(l+\frac{3}{2}-y\right) \frac{d}{d y}-\frac{1}{2}\left(l+\frac{3}{2}+\frac{\lambda}{2}\right)\right] \Gamma(y)=0 .
$$

The solutions of this equation, which guarantee that $\lim _{y \rightarrow \infty} g_{\kappa}(y)=0$, are the generalized Laguerre polynomials of degree $n, L_{n}^{p}(y)$, where

$$
\begin{gathered}
n=-\frac{1}{2}\left(l+\frac{3}{2}+\frac{\lambda}{2}\right), \\
p=l+\frac{1}{2} .
\end{gathered}
$$

From Eqs. (26) and (20)-(22), we can get immediately the eigenenergies, which are discrete since $n$ is an integer greater than or equal to zero:

$$
\begin{aligned}
\mathcal{E}^{2}- & m^{2}-(2 \kappa-1) m \omega_{2} \\
& =\left(2 n+l+\frac{3}{2}\right) \sqrt{2 m(\mathcal{E}+m) \omega_{1}^{2}+4 m^{2} \omega_{2}^{2}} .
\end{aligned}
$$

If we square Eq. (28) the resulting algebraic equation is quartic in $\mathcal{E}$. For particular values of $n$ and $\kappa$ (remember that $\kappa$ determines uniquely $l$ ) we can solve this equation with respect to $\mathcal{E}$ and get the energy of the level with quantum numbers $(n, \kappa)$ or, using the standard spectroscopic notation, the level $n l_{j}$. With the help of the program MATHEMATICA we were able to get analytical expressions for those solutions. In general, it can be shown that there are at most two real bound state solutions of Eq. (28), one with a positive or zero binding energy and the other one with a negative binding energy. As we will see later, it can happen that only positive binding energy solutions exist. When both solutions exist, we consider the one with positive binding energy, i.e., for which $\mathcal{E}>m$.

Figure 1 displays the energy for some levels, as a function of $\omega_{2}$, for a fixed $\omega_{1}$ and $m$. From this figure we see that for $\omega_{2}=0$ the energy levels with $(n=1, l=2)$ are degenerate with the level with $(n=2, l=0)$ and have no spin-orbit splitting. This is a manifestation of the $(2 n+l)$ degeneracy of the lev- 


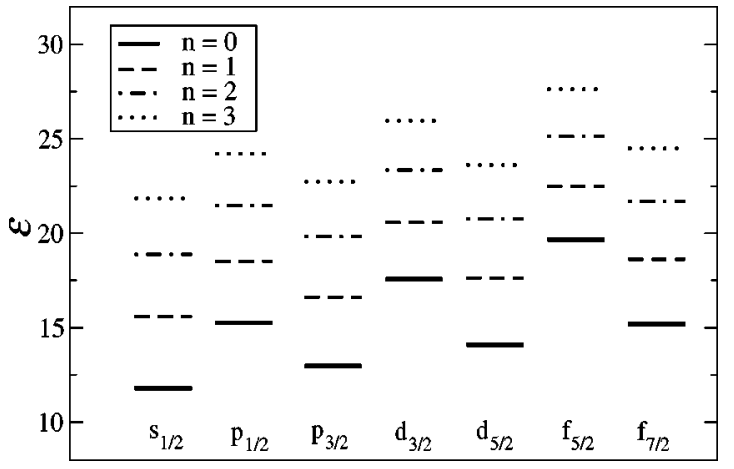

FIG. 2. Energy spectrum for $\Delta=0$ with $\omega_{1}=2, \omega_{2}=1$, and $m$ $=10$.

els which can be inferred from Eq. (28) when $\omega_{2}$ is set to zero. We will further elaborate on this point in the following section.

The spectrum for the first seven levels for $\omega_{2}=2$ and $\omega_{1}$ $=1$ is shown in Fig. 2, where we see that they are, in general, nondegenerate.

This feature of the spectrum can be understood by looking at Eq. (28), since when the tensor potential is present $\left(\omega_{2}\right.$ $\neq 0$ ) we have always a $\kappa$-dependent term (a spin-orbit term) that removes the $(2 n+l)$ degeneracy, which is also characteristic of the nonrelativistic harmonic-oscillator spectrum.

It is instructive to get the nonrelativistic limit of Eq. (28). We obtain this limit by letting $\omega_{1} / m$ and $\omega_{2} / m$ become very small. In this limit, $(\mathcal{E}-m) / m=E / m$ also becomes very small, and $\mathcal{E}+m \sim 2 m$, so that

$$
E=\left(2 n+l+\frac{3}{2}\right) \sqrt{\omega_{1}^{2}+\omega_{2}^{2}}+\left(\kappa-\frac{1}{2}\right) \omega_{2} .
$$

The solution of Eq. (19), replacing $y$ by $a^{2} r^{2}$, is

$$
g_{\kappa}(r)=A \exp \left(-\frac{1}{2} a^{2} r^{2}\right)\left(a^{2} r^{2}\right)^{(l+1) / 2} L_{n}^{l+1 / 2}\left(a^{2} r^{2}\right) \text {. }
$$

For the lower component, the first order differential equation in Eq. (13) allows us to write

$$
f_{\kappa}(r)=\frac{1}{\mathcal{E}+m}\left[\frac{d}{d r}+\frac{\kappa}{r}+m \omega_{2} r\right] g_{\kappa}(r) .
$$

Using the recursion relations for the generalized Laguerre polynomials (see, for example, Ref. [17]) we get, for $\kappa<0$,

$$
\begin{aligned}
f_{\kappa}(r)= & \frac{A a}{\mathcal{E}+m} \exp \left(-\frac{1}{2} a^{2} r^{2}\right)\left(a^{2} r^{2}\right)^{(l+2) / 2}\left[\left(\frac{m \omega_{2}}{a^{2}}+1\right)\right. \\
& \left.\times L_{n}^{l+1 / 2}\left(a^{2} r^{2}\right)-2 L_{n}^{l+3 / 2}\left(a^{2} r^{2}\right)\right]
\end{aligned}
$$

and, for $\kappa>0$,

$$
\begin{aligned}
f_{\kappa}(r)= & \frac{A a}{\mathcal{E}+m} \exp \left(-\frac{1}{2} a^{2} r^{2}\right)\left(a^{2} r^{2}\right)^{1 / 2}\left[\left(n+l+\frac{1}{2}\right)\left(1+\frac{m \omega_{2}}{a^{2}}\right)\right. \\
& \left.\times L_{n}^{l-1 / 2}\left(a^{2} r^{2}\right)+(n+1)\left(1-\frac{m \omega_{2}}{a^{2}}\right) L_{n+1}^{l-1 / 2}\left(a^{2} r^{2}\right)\right] .
\end{aligned}
$$

Since the generalized Laguerre polynomials of degree $n$ have $n$ distinct zeros [17] we may conclude that $g_{\kappa}$ has $n$ nodes, and the expression for $f_{\kappa}$ suggests that it has $n$ nodes for $\kappa<0$ and $n+1$ for $\kappa>0$,

$$
n_{f}=\left\{\begin{array}{l}
n_{g}, \quad \kappa<0 \\
n_{g}+1, \quad \kappa>0 .
\end{array}\right.
$$

This is indeed verified by the radial functions for the states $2 s_{1 / 2}, 1 d_{3 / 2}$, and $1 d_{5 / 2}$ plotted in Fig. 3 .

Next, let us consider the other case

$$
\Sigma(r)=0, \Delta(r)=\frac{1}{2} m \omega_{1}^{2} r^{2}, \quad U(r)=m \omega_{2} r,
$$

where $\omega_{2}$ is again the frequency related to the tensor potential $U$, and $\omega_{1}$ is the frequency now related to the "difference" potential, $\Delta$. We now start from the equation for the lower component, Eq. (17), and introduce the new variable and parameters defined by

$$
\begin{gathered}
\tilde{y}=\sqrt{\frac{m(\mathcal{E}-m)}{2}} \tilde{\Omega} r^{2}=\tilde{a}^{2} r^{2}, \\
\tilde{\Omega}=\sqrt{\frac{2 m}{\mathcal{E}-m} \omega_{2}^{2}+\omega_{1}^{2},} \\
\tilde{\lambda}=\frac{(2 \kappa+1) m \omega_{2}-\left(\mathcal{E}^{2}-m^{2}\right)}{\tilde{a}^{2}} .
\end{gathered}
$$

The resulting equation for $f_{\kappa}$ in these variables is formally the same as Eq. (23). Following the same steps as before, to guarantee that $f_{\kappa}(r)$ vanishes when $r \rightarrow \infty$, we must have

$$
\tilde{n}=-\frac{1}{2}\left(\tilde{l}+\frac{3}{2}+\frac{\tilde{\lambda}}{2}\right)
$$

where $\tilde{n}$ is an integer greater than or equal to zero and $\tilde{l}$ is given by Eq. (11). This last quantum number is called pseudoorbital angular momentum quantum number in view of its role in the pseudospin symmetry, which will be discussed in Sec. IV. Similarly to Eq. (10) for $\kappa$, we may define $\widetilde{\kappa}$ in terms of $\tilde{l}$ and $\tilde{s}=1 / 2$ (pseudospin quantum number):

$$
\tilde{\kappa}=\left\{\begin{array}{r}
-(\tilde{l}+1)=-(j+1 / 2), \quad j=\tilde{l}+1 / 2 \quad(\widetilde{\kappa}<0) \\
\text { aligned pseudospin } \\
\tilde{l}=+(j+1 / 2), \quad j=\tilde{l}-1 / 2 \quad(\tilde{\kappa}>0) \\
\text { unaligned pseudospin } .
\end{array}\right.
$$

In other words, $\widetilde{\kappa}=-\kappa$ is a parameter describing the coupling between the pseudo-orbital angular momentum and the pseudospin.

From Eq. (39) we get the following equation for the energy: 

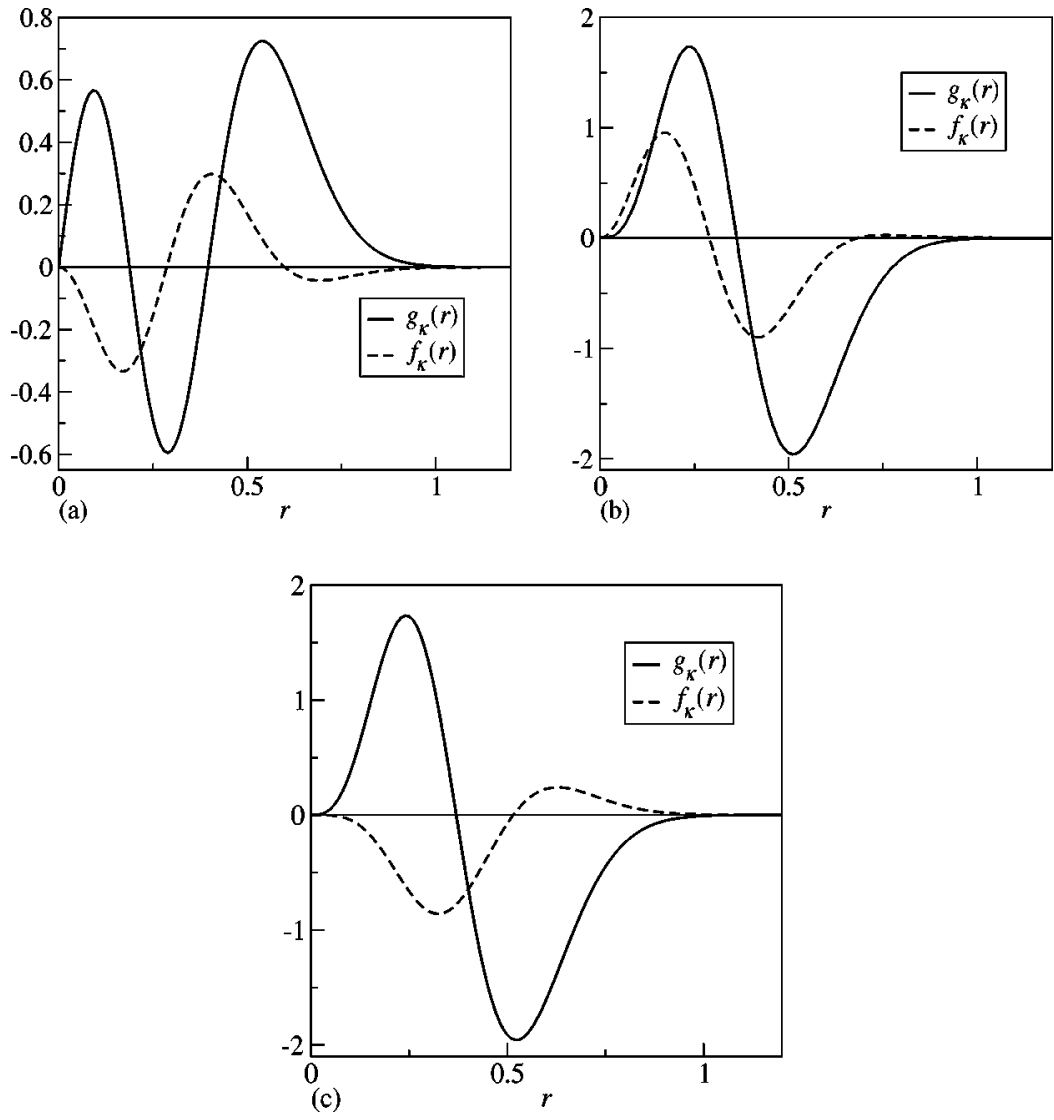

$$
\begin{aligned}
\mathcal{E}^{2}- & m^{2}+(2 \widetilde{\kappa}-1) m \omega_{2} \\
& =\left(2 \tilde{n}+\tilde{l}+\frac{3}{2}\right) \sqrt{2 m(\mathcal{E}-m) \omega_{1}^{2}+4 m^{2} \omega_{2}^{2}} .
\end{aligned}
$$

Again, there are at most two real solutions of this equation. For positive $\omega_{2}$ and for most cases when $\omega_{2}<0$, it can be shown that there is always a positive binding energy solution. This is the solution we will take in the following examples. Some energy levels for $\omega_{1}=2$ and $\omega_{2}$ in a range from 0 to 1 are shown in Fig. 4. For $\omega_{2}=0$ there is a $(2 \tilde{n}+\tilde{l})$ degeneracy of what we might call a pseudoharmonic oscillator. For a nonvanishing $\omega_{2}$ this degeneracy is removed as we can also see in Fig. 5. In these figures we use the spectroscopic notation $\tilde{n} \widetilde{l}_{j}$, where states with $\tilde{l}=0,1,2, \ldots$ are denoted by $\tilde{s}, \tilde{p}, \tilde{d}, \ldots$, respectively. The ground state for $\omega_{1}$ $=2$ and $\omega_{2}=0$ is the state $\tilde{0} \widetilde{s}_{1 / 2}$, but we see from Fig. 4 that it is replaced by the state $\tilde{0} \tilde{p}_{1 / 2}$ as the ground state when $\omega_{1}$ $=2$ and $\omega_{2} \gtrsim 0.3$.

The nonrelativistic limit of the eigenvalue equation in Eq. (41) is again reached by decreasing $\omega_{1}$ and $\omega_{2}$ such that $\omega_{1} / m \ll 1$ and $\omega_{2} / m \ll 1$. If we solve the quartic eigenvalue equation for $E / m$ and expand it in powers of $\omega_{1} / \mathrm{m}$ and $\omega_{2} / \mathrm{m}$ we get, in lowest order of these expansion parameters,
FIG. 3. Radial wave functions for $\Delta=0$ of the states (a) $2 s_{1 / 2}$, (b) $1 d_{3 / 2}$, and (c) $1 d_{5 / 2}$ with $\omega_{1}$ $=2, \omega_{2}=1$, and $m=10$.

$$
\begin{aligned}
E= & \left(2 \tilde{n}+\tilde{l}+\frac{3}{2}\right)\left|\omega_{2}\right|-\left(\tilde{\kappa}-\frac{1}{2}\right) \omega_{2}+\frac{1}{4 m}\left(2 \tilde{n}+\tilde{l}+\frac{3}{2}\right)[2 \tilde{n}+\tilde{l} \\
& \left.+\frac{3}{2}-\operatorname{sign}\left(\omega_{2}\right)\left(\widetilde{\kappa}-\frac{1}{2}\right)\right] \omega_{1}^{2} .
\end{aligned}
$$

This equation, valid for $\Sigma=0$ and $\omega_{2} \neq 0$, shows that the effect of the harmonic-oscillator $\Delta$ potential is only of second order in a nonrelativistic expansion. This important re-

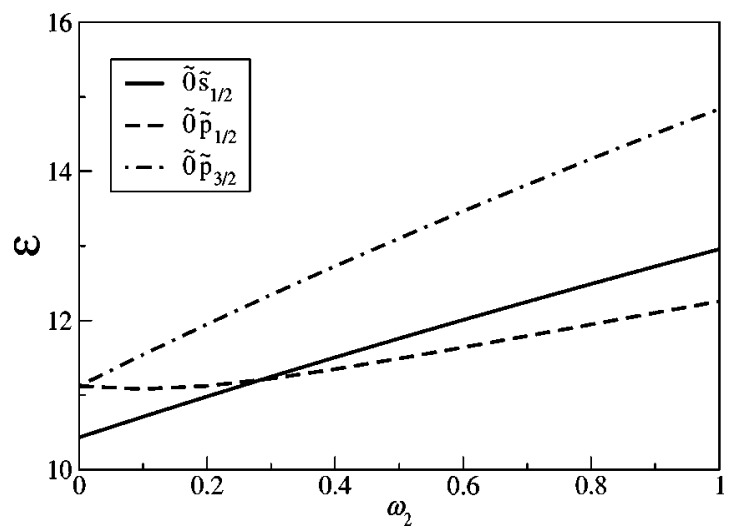

FIG. 4. Energy levels for $\Sigma=0$ with $\omega_{1}=2$ and $m=10$ as a function of $\omega_{2}$. 


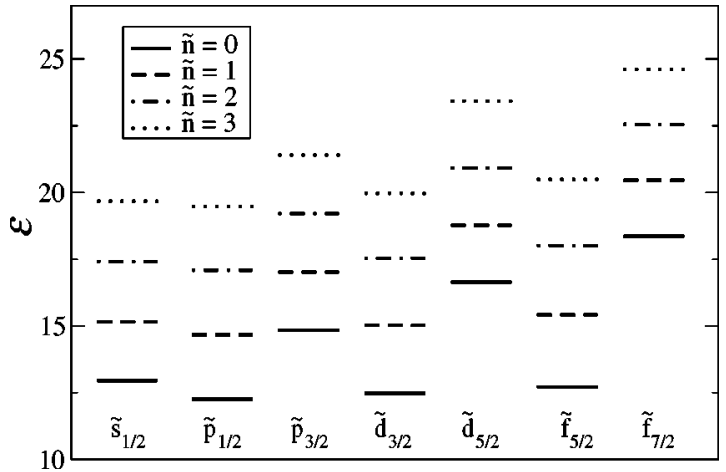

FIG. 5. Energy spectrum for $\Sigma=0$ with $\omega_{1}=2, \omega_{2}=1$, and $m$ $=10$.

sult sheds light on the relativistic nature of the pseudospin symmetry to be discussed later in this paper.

A comparison between Figs. 1 and 4 shows that the effect of the tensor potential (seen by the variation of $\omega_{2}$ ), is bigger for the case $\Sigma=0$ than is for $\Delta=0$. As a consequence, we still see in Fig. 5 a quasidegeneracy for some levels, in particular, those with the same value of $\tilde{n}$ and that have $\tilde{l}=\widetilde{\kappa}$ (aligned spin). This can be explained by the energy dependence on $\omega_{1}^{2} / m$ in Eq. (42) (for small values of $\omega_{1} / m$ and $\omega_{2} / m$ ), a number smaller than $\omega_{2}$, meaning that in this case we are near the usual Dirac oscillator $\left(\omega_{1}=0\right.$ and $\left.\omega_{2} \neq 0\right)$, where the states with aligned $\operatorname{spin}(j=l+1 / 2)$ and the same $n$ are all degenerate, as we will discuss in the following section.

The lower component radial wave function is

$$
f_{\kappa}(r)=B \exp \left(-\frac{1}{2} \widetilde{a}^{2} r^{2}\right)\left(\widetilde{a}^{2} r^{2}\right)^{(\tilde{l}+1) / 2} L_{\widetilde{n}}^{\tilde{l}+1 / 2}\left(\widetilde{a}^{2} r^{2}\right)
$$

where $B$ is again a normalization constant, determined by Eq. (8). As already commented, the degree of the generalized Laguerre polynomial determines its number of zeros, so that $\tilde{n}$ gives the number of nodes in $f_{\kappa}$.

The upper component for the set of potentials in Eq. (35) is obtained from the first order differential equation in Eq. (14), i.e.,

$$
g_{\kappa}(r)=-\frac{1}{\mathcal{E}-m}\left[\frac{d}{d r}+\frac{\tilde{\kappa}}{r}-m \omega_{2} r\right] f_{\kappa}(r),
$$

whose explicit form is given, using again the recursion relations of the Laguerre polynomials, for $\tilde{\kappa}<0$, by

$$
\begin{aligned}
g_{\kappa}(r)= & -\frac{B \tilde{a}}{\mathcal{E}-m} \exp \left(-\frac{1}{2} \widetilde{a}^{2} r^{2}\right)\left(\tilde{a}^{2} r^{2}\right)^{(\tilde{l}+2) / 2}\left[\left(1-\frac{m \omega_{2}}{\tilde{a}^{2}}\right)\right. \\
& \left.\times L_{\tilde{n}}^{\tilde{l}+1 / 2}\left(\widetilde{a}^{2} r^{2}\right)-2 L_{\tilde{n}}^{\tilde{l}+3 / 2}\left(\tilde{a}^{2} r^{2}\right)\right],
\end{aligned}
$$

and, for $\tilde{\kappa}>0$,

$$
\begin{aligned}
g_{\kappa}(r)= & -\frac{B \tilde{a}}{\mathcal{E}-m} \exp \left(-\frac{1}{2} \widetilde{a}^{2} r^{2}\right)\left(\tilde{a}^{2} r^{2}\right)^{\tilde{l} / 2}\left[\left(\tilde{n}+\tilde{l}+\frac{1}{2}\right)\right. \\
& \times\left(1-\frac{m \omega_{2}}{\tilde{a}^{2}}\right) L_{\tilde{n}}^{\tilde{l}-1 / 2}\left(\widetilde{a}^{2} r^{2}\right)+(\tilde{n}+1) \\
& \left.\times\left(1+\frac{m \omega_{2}}{\tilde{a}^{2}}\right) L_{\tilde{n}+1}^{\tilde{l}-1 / 2}\left(\widetilde{a}^{2} r^{2}\right)\right] .
\end{aligned}
$$

These functions are analogous to the $g_{\kappa}$ and $f_{\kappa}$ functions of the previous case, so from the above discussion we can conclude that the number of nodes of the radial functions for $\Sigma=0$ is such that (remember that $\tilde{\kappa}=-\kappa$ )

$$
n_{f}=\left\{\begin{array}{l}
n_{g}-1, \quad \kappa<0 \\
n_{g}, \quad \kappa>0 .
\end{array}\right.
$$

This is illustrated in the plots of $g_{\kappa}$ and $f_{\kappa}$ in Fig. 6. Adopting the definition of $\tilde{n}$ as the number of nodes of the lower component when $\omega_{2}=0$ and $\Sigma=0$, we see that we can convert from the notation $n l_{j}$ to $\tilde{n}_{j}$ for the same state by setting $n \rightarrow \tilde{n}=n, l \rightarrow \tilde{l}=l-1$ when $\kappa>0$ and $n \rightarrow \tilde{n}=n-1, l$ $\rightarrow \tilde{l}=l+1$ when $\kappa<0$.

We have noticed, however, that the radial function node structure depends on the ratio $\omega_{1} / \omega_{2}$. If $\omega_{2}$ becomes significantly larger than $\omega_{1}$, in the case of $\Delta=0$ the structure of the nodes is the one of the normal Dirac oscillator, the same as in Eq. (47), as it will be discussed in the following section.

\section{PARTICULAR CASES}

Now we will present some results for the three particular cases $\omega_{1}=0, \omega_{2}=0\left(\Delta=0\right.$ and $\left.\sum=0\right)$

\section{A. Case $\omega_{1}=0$}

As mentioned before, the expression "Dirac oscillator" applies to this case when $\omega_{2}>0$ [2]. Here we also consider the possibility that $\omega_{2}$ be negative. From Eq. (19) when $\omega_{1}$ $=0$, it is straightforward to conclude that the equation for the upper radial wave function of the Dirac oscillator is a Schrödinger-like equation with a harmonic-oscillator potential and a spin-orbit coupling term.

The eigenenergies are readily obtained taking the limit $\omega_{1} \rightarrow 0$ in Eq. (28), which yields

$$
\mathcal{E}^{2}=m^{2}+m\left|\omega_{2}\right|\left[2\left(N+\frac{3}{2}\right)+\operatorname{sgn}\left(\omega_{2}\right)(2 \kappa-1)\right],
$$

where the quantum number $N=2 n+l$ was introduced. There are, in general (when the right-hand side of the equation is positive) two symmetric real solutions for this equation. Here we take the positive energy solution. Note that Eq. (48) results from the equation for the upper component, $g_{\kappa}$. We can get the nonrelativistic energy by setting $\omega_{1}=0$ in Eq. (29). Therefore, in this limit we obtain for the energy $E$ the quantum mechanical result $\left|\omega_{2}\right|(N+3 / 2)$ plus the spin-orbit contribution $\frac{1}{2}(2 \kappa-1) \omega_{2}$. This term removes the nonrelativistic degeneracy related to levels with the same $N$ in such a way that changing the sign of $\omega_{2}$ leads to a order reversal of the 

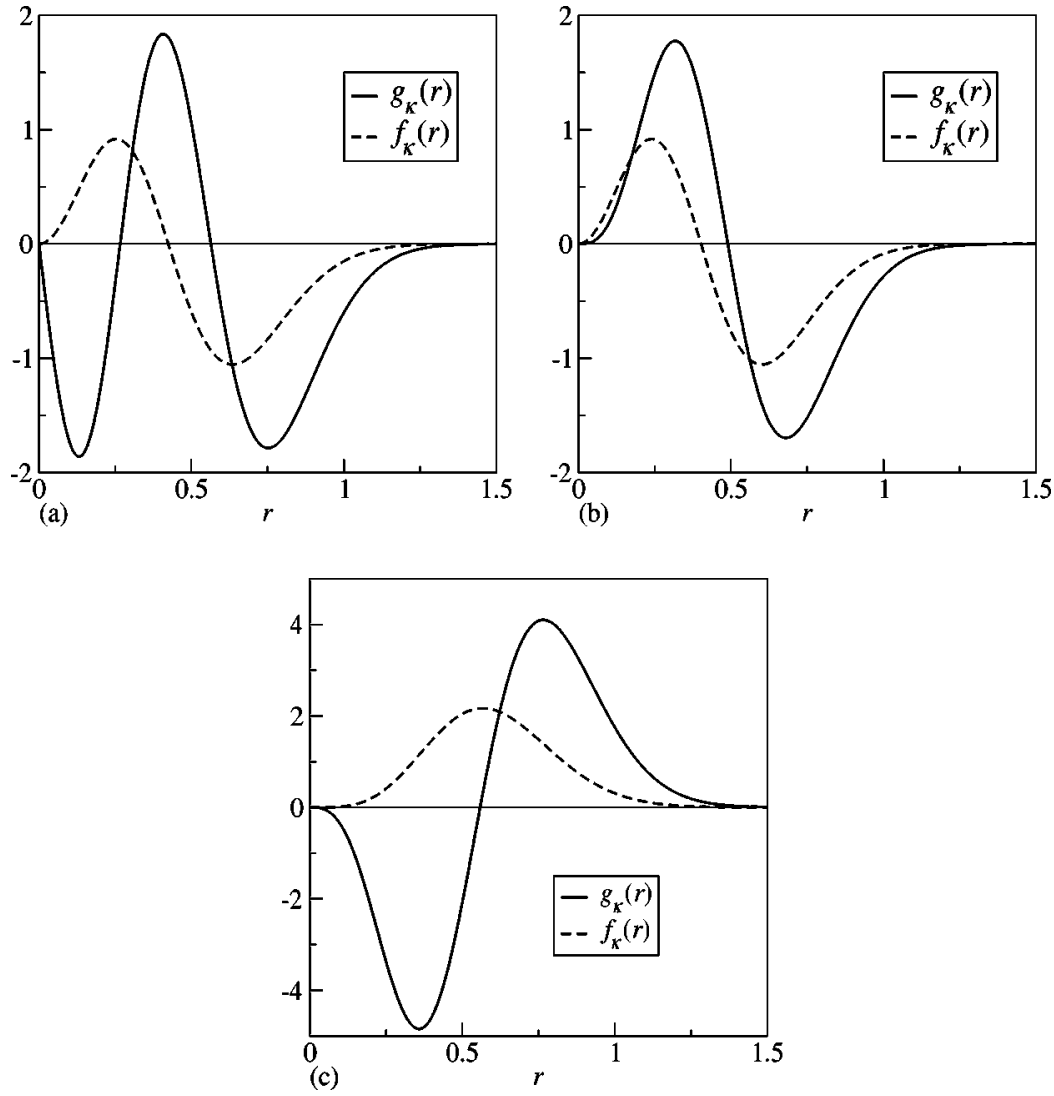

levels with the same $(n, l)$ and $j=l \pm 1 / 2$ quantum numbers. For $\omega_{2}>0$, depending on whether the quantum number $\kappa$ is positive or negative, the energy is given by [see Eq. (10)]

$$
\begin{gathered}
\mathcal{E}^{2}=m^{2}+4 m \omega_{2}\left(n+l+\frac{1}{2}\right), \quad \kappa>0, \\
\mathcal{E}^{2}=m^{2}+4 m \omega_{2} n, \quad \kappa<0 .
\end{gathered}
$$

Thus, for a given quantum number $n$, all states with aligned spin $j=l+1 / 2(\kappa<0)$ and arbitrary orbital angular momentum are degenerate. This degeneracy, as well as the $(n+l)$ degeneracy for $\kappa>0$, is illustrated in Fig. 7 , in which the spectrum for $\omega_{2}=1$ is presented.

On the other hand, the eigenenergies of the Dirac oscillator Hamiltonian may also be determined from Eq. (41), which was obtained from the second order equation for the lower component $f_{\kappa}$, very similar to Eq. (19). Again taking the limit $\omega_{1} \rightarrow 0$ in Eq. (41) one obtains

$$
\mathcal{E}^{2}=m^{2}+m\left|\omega_{2}\right|\left[2\left(\tilde{N}+\frac{3}{2}\right)+\operatorname{sgn}\left(\omega_{2}\right)(2 \kappa+1)\right],
$$

where $\tilde{N}=2 \tilde{n}+\tilde{l}$ is a new quantum number. The term $2 \kappa+1$ comes from the spin-orbit interaction for the lower component. Depending on whether the quantum number $\kappa$ is positive or negative, the energy, for $\omega_{2}>0$, is given by

$$
\mathcal{E}^{2}=m^{2}+4 m \omega_{2}\left(\tilde{n}+\tilde{l}+\frac{3}{2}\right), \quad \kappa>0,
$$

FIG. 6. Radial wave functions for $\Sigma=0$ of the states (a) $\tilde{1}_{1 / 2}\left(2 s_{1 / 2}\right)$, (b) $\tilde{1} \widetilde{p}_{3 / 2}\left(1 d_{3 / 2}\right)$, and (c) $\tilde{0}_{5 / 2}\left(1 d_{5 / 2}\right)$ with $\omega_{1}=2, \omega_{2}=1$, and $m=10$.

$$
\mathcal{E}^{2}=m^{2}+4 m \omega_{2}(\tilde{n}+1), \quad \kappa<0 .
$$

Of course, the eingenvalue equations in Eqs. (52) and (53) should lead to the same energy values as Eqs. (49) and (50). Remembering that, for $\kappa<0$, one has $\tilde{l}=l+1$, and, for $\kappa>0$, $\tilde{l}=l-1$, from the comparison of those two sets of equations one concludes that

$$
n_{f}=\left\{\begin{array}{cc}
n_{g}-1, & \kappa<0 \\
n_{g}, & \kappa>0,
\end{array}\right.
$$

since the quantum numbers $n$ and $\tilde{n}$ are equal to the number of nodes of the upper and lower radial functions, denoted by

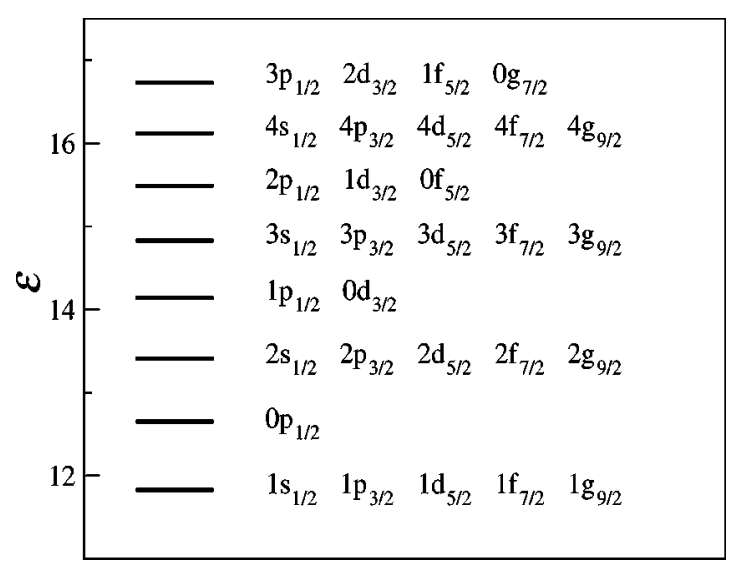

FIG. 7. Energy spectra for the normal Dirac oscillator $\left(\omega_{1}=0\right)$ with $\omega_{2}=1$ and $m=10$. 


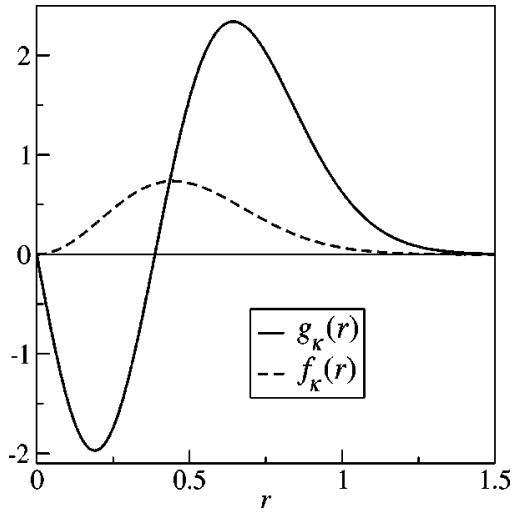

FIG. 8. Ground state radial wave functions for the normal Dirac oscillator with the same parameters as in Fig. 7.

$n_{g}$ and $n_{f}$, respectively. Notice that $n_{g} \geqslant 1$ for $\kappa<0$. This can also be inferred from the analytical form of the radial upper and lower functions for the Dirac oscillator. These components are readily obtained from Eqs. (30), (32), and (33), noticing that, when $\omega_{1}=0$, the square of the parameter $a$, defined in Eq. (22), is equal to $m \omega_{2}\left(\omega_{2}>0\right)$, giving

$$
g_{\kappa}(r)=A \exp \left(-\frac{1}{2} a^{2} r^{2}\right)\left(a^{2} r^{2}\right)^{(l+1) / 2} L_{n}^{l+1 / 2}\left(a^{2} r^{2}\right)
$$

for the upper component, and

$$
\begin{aligned}
f_{\kappa}(r)= & -\frac{2 A a}{\mathcal{E}+m} \exp \left(-\frac{1}{2} a^{2} r^{2}\right) \\
& \times\left(a^{2} r^{2}\right)^{(l+2) / 2} L_{n-1}^{l+3 / 2}\left(a^{2} r^{2}\right) \quad(\kappa<0), \\
f_{\kappa}(r)= & \frac{2 A a}{\mathcal{E}+m} \exp \left(-\frac{1}{2} a^{2} r^{2}\right)\left(a^{2} r^{2}\right)^{1 / 2}\left(n+l+\frac{1}{2}\right) \\
& \times L_{n}^{l-1 / 2}\left(a^{2} r^{2}\right) \quad(\kappa>0)
\end{aligned}
$$

for the lower component [we have used the relation $\left.L_{n}^{\alpha-1}(x)-L_{n}^{\alpha}(x)=-L_{n-1}^{\alpha}(x)[17]\right]$.

As we have seen before, the degree of these Laguerre polynomials is identified with the principal quantum number of the upper and lower radial functions, and thus with their number of nodes, so that the relations in Eq. (54) follow immediately.

In this case $\left(\omega_{2}>0\right)$ Eq. (54) implies a peculiar node structure for the ground state $s_{1 / 2}(\kappa=-1)$. This state has a node $\left(n_{g}=1\right)$ in the upper component, as we can see in Fig. 8, whereas the nonrelativistic ground state wave function is nodeless.

If $\omega_{2}<0$, the infinite degeneracy referred to above occurs for $\kappa>0$, i.e., for nonaligned spins. In addition, the relation between the values $n_{g}$ and $n_{f}$ is modified: $n_{f}=n_{g}+1$ for $\kappa>0$ and $n_{f}=n_{g}$ for $\kappa<0$. It is worth noticing that the structure of the radial nodes for $\omega_{2}$ positive (negative) is exactly the same for the case $\Sigma=0(\Delta=0)$ discussed in the preceding section. This same node structure could again be inferred from the form of the radial wave functions when $\omega_{2}<0$, which can be obtained once more from Eqs. (30), (32), and (33), setting $a^{2}=-m \omega_{2}$. We note that now the ground state $s_{1 / 2}$ has zero nodes in the upper component, and therefore, in the nonrelativistic limit, when the lower component disappears, the wave function has the same node structure as the nonrelativistic harmonic-oscillator ground state wave function. Furthermore, from Eq. (51) we see that, for $\omega_{2}<0$, the sign of the spin-orbit interaction is inverted, such that states with nonaligned spin $(\kappa>0)$ have now lower energy than states with the same $l$ and aligned spin. This is the situation in atomic physics, where, for example, the states $p_{3 / 2}$ have higher energy than the states $p_{1 / 2}$. The opposite happens in nuclear physics, where states with aligned spin are lower in energy than nonaligned spin states, which in the present case happens when $\omega_{2}>0$.

We present in Fig. 9 the radial wave functions for the states $2 s_{1 / 2}, 1 d_{3 / 2}$, and $1 d_{5 / 2}$. From these plots we see that the upper components for the spin-orbit partners (same $n, l$ ) are equal [see Fig. 9(b) and 9(c), since both are given by the same radial wave function, Eq. (55). For pairs with the same $\tilde{l}$ (pseudospin partners) the lower components are the same, up to a constant factor. This can be seen from Eqs. (57) and (56), since the pseudospin partners have quantum numbers $(n, l)(\kappa<0)$ and $(n-1, l+2)(\kappa>0)$. This particular behavior could be related to a particular symmetry of the Dirac Hamiltonian in Eq. (2) with $\omega_{1}=0$, as is the case with the pseudospin symmetry, to be discussed in Sec. IV.

\section{B. Case $\omega_{2}=0$ and $\Delta=0$}

In this subsection we set to zero both $\Delta$ and $U$ potentials. Then, Eq. (19) with $\omega_{2}=0$ leads to the following second order differential equation for the upper component [note that $\kappa(\kappa+1)=\ell(\ell+1)$, irrespective of the sign of $\kappa]$ :

$$
\left[\frac{d^{2}}{d r^{2}}-\frac{l(l+1)}{r^{2}}-\frac{m(\mathcal{E}+m)}{2} \omega_{1}^{2} r^{2}-\left(m^{2}-\mathcal{E}^{2}\right)\right] g_{\kappa}(r)=0 .
$$

The solutions of this equation are given by Eq. (30), where $a$ is given by Eqs. (20)-(22) in which $\omega_{2}$ is set to zero. The upper and lower radial functions for two levels corresponding to $\kappa$ with opposite signs are plotted in Fig. 10. From this figure we see that the node structure is the same as given in the preceding section when $\omega_{2} \neq 0$ [Eq. (34)].

The energy eigenvalues are readily obtained from Eq. (28) by just taking the limit $\omega_{2} \rightarrow 0$, yielding

$$
(\mathcal{E}-m) \sqrt{\frac{\mathcal{E}+m}{2 m}}=\omega_{1}\left(2 n+l+\frac{3}{2}\right) \quad(n=0,1,2, \ldots) .
$$

This equation for the energy, valid for $\Delta=0$, shows explicitly that there is no spin-orbit term and that states with $j$ $=l \pm 1 / 2$ are degenerate. We can prove that this equation has only one real solution, and it has to be such that $\mathcal{E}>m$. To begin with, since the right-hand side is always positive, it is clear that any real solution must be greater than $m$, otherwise the left-hand side would be negative. We prove that there is only one of such solutions by squaring Eq. (59) and using the Descartes' rule of signs. This rule states that the number of 

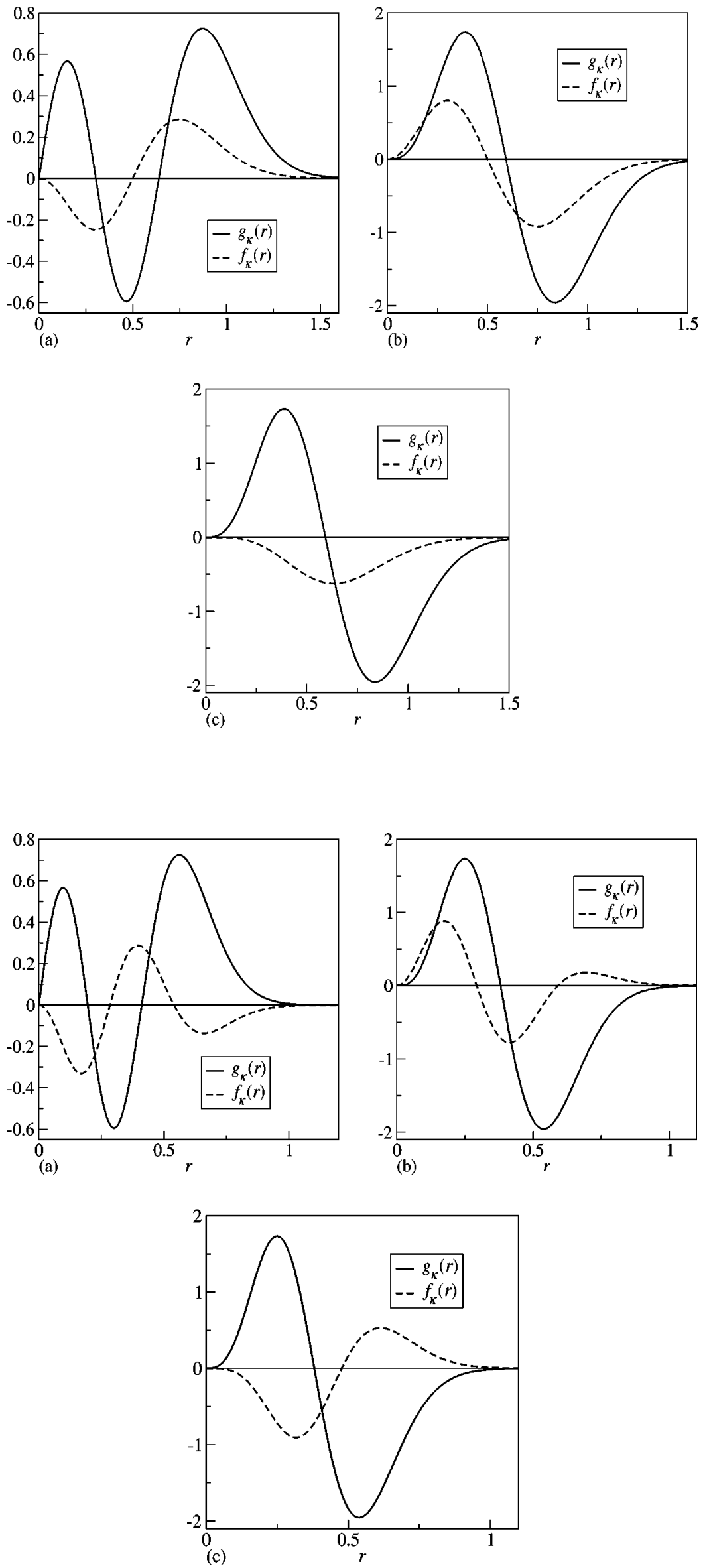

FIG. 9. Radial wave functions for the normal Dirac oscillator of the states (a) $2 s_{1 / 2}$, (b) $1 d_{3 / 2}$, and (c) $1 d_{5 / 2}$ with the same parameters as in Fig. 7.
FIG. 10. Radial wave functions for the potential $\Sigma(r)=\frac{1}{2} m \omega_{1}^{2} r^{2}$, with $U=\Delta=0$ for the states $2 s_{1 / 2}, 1 d_{3 / 2}$, and $1 d_{5 / 2}$, respectively, with $\omega_{1}=2$ and $m=10$. 


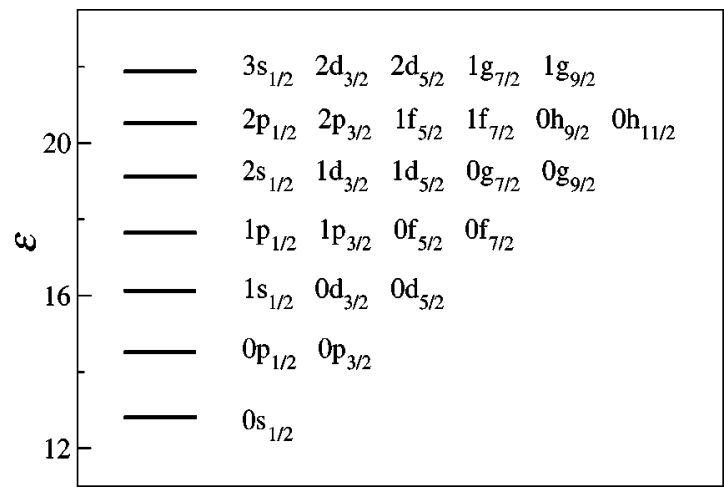

FIG. 11. Single particle energies for the case $\Delta=U=0$ with $\omega_{1}$ $=2, \omega_{2}=0$, and $m=10$.

positive real roots of an algebraic equation with real coefficients $a_{k} x^{k}+\cdots+a_{1} x+a_{0}=0$ is never greater than the number of changes of signs in the sequence $a_{k}, \ldots, a_{1}, a_{0}$ (not counting the null coefficients) and, if less, then always by an even number $[26,27]$. Since we can write the square of Eq. (59) as

$$
x^{3}+2 x^{2}-a^{2}=0,
$$

with $x=(\mathcal{E}-m) / m$ and $a=\sqrt{2} \omega_{1}(2 n+l+3 / 2) / m$, then, by Descartes' rule of signs, only one solution with $x>0$, i.e., $\mathcal{E}>m$, exists. Since by squaring Eq. (59) we may only introduce a negative $(x<0)$ spurious solution, this solution is the only positive solution of that equation, and the proof is complete. Therefore, for given values of $\omega_{1}, n$ and $l$, i.e, only discrete, positive (greater than $m$ ) energies are allowed.

The nonrelativistic limit is obtained by enforcing $\omega_{2} \rightarrow 0$ in Eq. (29). The right-hand side becomes the expression for the nonrelativistic energy eigenvalues of the harmonic oscillator with no spin-orbit coupling. Similarly, Eq. (30) gives the nonrelativistic harmonic-oscillator radial wave function, with $a^{2}=m \omega_{1}$. Figure 11 shows the single particle spectrum for $\Delta=0, m=10$, and $\omega_{1}=2$. For sufficiently small $\omega_{1}$ the energy levels are essentially equidistant, a well-known feature of nonrelativistic harmonic-oscillator energy levels, and we do not have spin-orbit interaction: states with $j=l \pm 1 / 2$ are degenerate. These results allow us to stress that the $\Delta$ potential can be closely connected to the intrinsic relativistic content of a theory with scalar $(S)$ and vector $(V)$ potentials. The nonrelativistic harmonic oscillator is in fact the limit of the relativistic theory with $\Delta=V-S=0$ and a potential well $\Sigma=S+V$ when $\omega_{1} / m$ becomes very small.

Thus, an important conclusion of our study is that a Dirac equation with scalar and vector harmonic-oscillator potentials with the same sign and magnitude $(\Delta=0)$, instead of a linear tensor potential $U$, seems to be the most natural way to introduce the harmonic oscillator in relativistic quantum mechanics.

\section{Case $\omega_{2}=0$ and $\Sigma=0$}

Now we consider that both the tensor potential $U$ and $\Sigma$ are zero. The situation is very similar to the preceding sub- section, except that we now find that it is the lower component that satisfies the following second order differential equation:

$$
\left[\frac{d^{2}}{d r^{2}}-\frac{\tilde{l}(\tilde{l}+1)}{r^{2}}-\frac{m(\mathcal{E}-m)}{2} \omega_{1}^{2} r^{2}-\left(m^{2}-\mathcal{E}^{2}\right)\right] f_{\kappa}(r)=0
$$

[note that $\kappa(\kappa-1)=\widetilde{\kappa}(\widetilde{\kappa}+1)=\widetilde{\ell}(\widetilde{\ell}+1)]$. The solution of this equation is the function (43) setting $\omega_{2}=0$ in Eq. (37).

These radial wave functions are represented in Fig. 12 for the $2 s_{1 / 2}, 1 d_{3 / 2}$, and $2 d_{5 / 2}$ states. Again, we see that the node structure is the same as the one given by Eq. (47). For the states $2 s_{1 / 2}$ and $1 d_{3 / 2}$, which are pseudospin doublets as we will see later, the lower components are equal. This is a general feature of pseudospin symmetry, since when one acts upon a given state with the pseudospin $\mathrm{SU}(2)$ generators to obtain its pseudospin partner, the radial lower component is not changed because of the particular form of these generators, as was shown in Refs. $[16,18]$.

The eigenenergies for $\Sigma=U=0$ are obtained taking the limit $\omega_{2} \rightarrow 0$ in Eq. (41), which leads to

$$
(\mathcal{E}+m) \sqrt{\frac{\mathcal{E}-m}{2 m}}=\omega_{1}\left(2 \tilde{n}+\tilde{l}+\frac{3}{2}\right) \quad(\tilde{n}=0,1,2, \ldots) .
$$

Again, since the right-hand side of Eq. (62) is positive and real, we see that the real solutions must have positive binding energy $\mathcal{E}=\mathcal{E}-m$. Using similar arguments as with Eq. (59), one can show that there can be just one of such solutions.

The nonrelativistic limit in this case is obtained by setting $\omega_{2}=0$ in Eq. (42). As remarked above, the resulting expression is of second order in $\omega_{1} / \mathrm{m}$, meaning that the energy is zero up to first order in $\omega_{1} / \mathrm{m}$. We can interpret this fact by saying that, up to this order, there is no nonrelativistic limit for $\Sigma=U=0$ and therefore the theory is intrinsically relativistic and so is the pseudospin symmetry. In this case, the second order equation in Eq. (61), which only depends on $\tilde{l}$, and also the eigenenergies in Eq. (62), show that there is no pseudospin-orbit coupling and therefore the states with same $(\tilde{n}, \widetilde{l})$, but with $j=\tilde{l}+1 / 2$ and $j=\tilde{l}-1 / 2$, are degenerate. Thus, when $\Delta$ is a harmonic-oscillator potential and $\Sigma=U=0$, there are only positive-energy bound states and exact pseudospin symmetry, i.e., $\Delta$ acts as a binding potential. This is an interesting result in view of the fact that the pseudospin symmetry obtained in the limit $\Sigma(r) \rightarrow 0$ cannot be realized for nuclear vector and scalar mean fields which go to zero as $r$ $\rightarrow \infty$, since in that case $\Sigma$ acts as a binding negative central potential well and therefore no bound states may exist when $\Sigma=0[12,13]$. The spectrum of single particle states for the case $\Sigma=0$ and $m=10$ is shown in Fig. 13(a) using the quantum numbers of the upper component, which can be seen as the analog of the nonrelativistic quantum numbers. In Fig. 13(b) we classify the same energy levels by the quantum numbers of the lower components $f_{\kappa}$. The comparison between these two figures manifests the pseudospin symmetry and its quantum numbers. For example, the doublets $\left[1 s_{1 / 2}\right.$ 

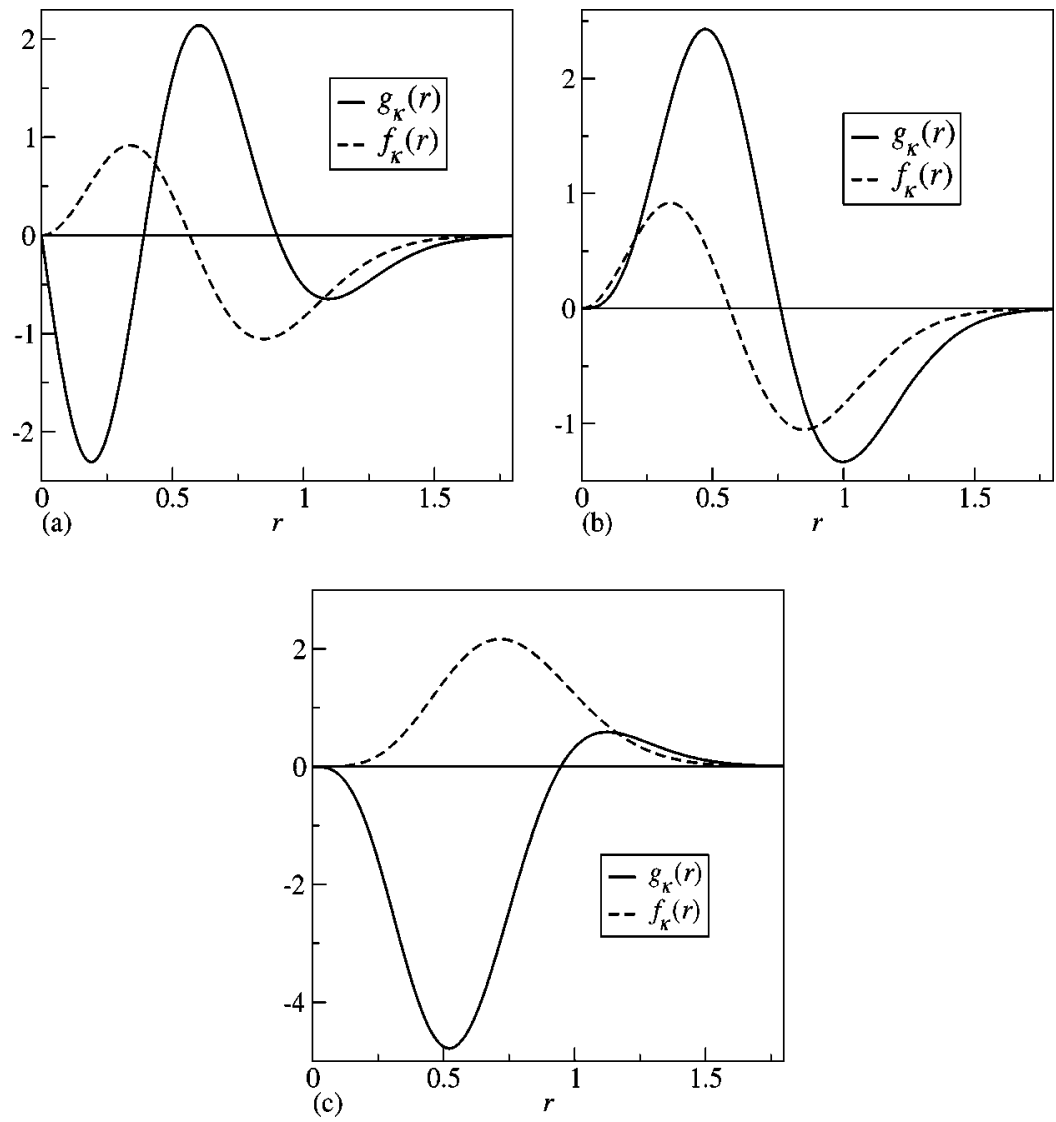

FIG. 12. Radial wave functions for the potential $\Sigma=0$ of the states (a) $\tilde{1} \tilde{p}_{1 / 2}\left(2 s_{1 / 2}\right)$, (b) $\tilde{1}_{\tilde{p}_{3 / 2}}\left(1 d_{3 / 2}\right)$, and (c) $\tilde{0}_{5 / 2}\left(1 d_{5 / 2}\right)$ with the same parameters as in Fig. 11.

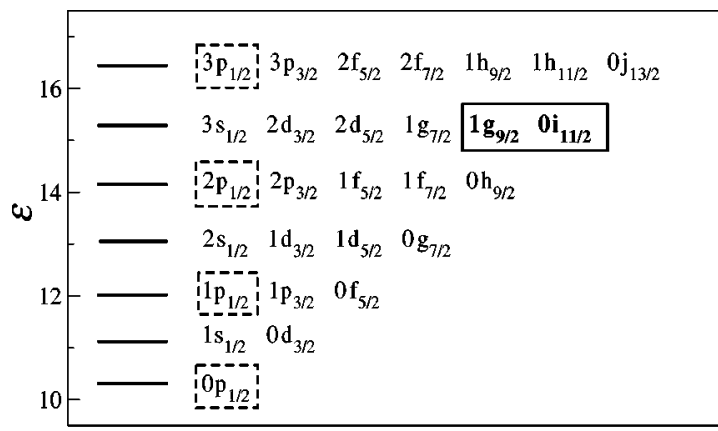

(b)

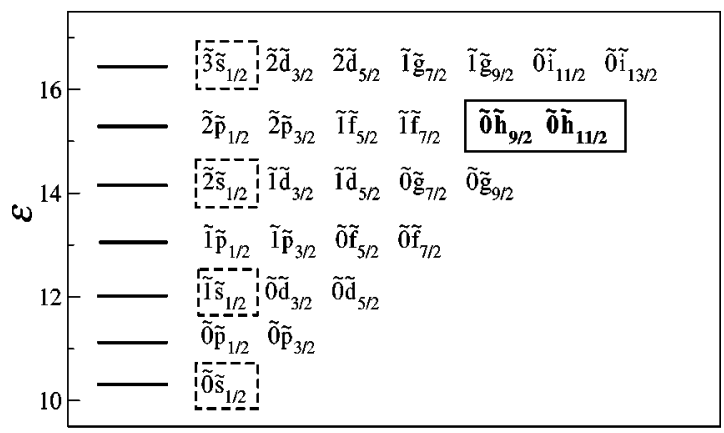

(b)

FIG. 13. Single particle energies for the case $\Sigma=0$ with the quantum numbers (a) $(n l j)$ and (b) $(\widetilde{n} \widetilde{l j}=j)$. The parameters are $\omega_{1}=2, \omega_{2}=0$, and $m=10$. $\left.-0 d_{3 / 2}\right]$ and $\left[1 p_{3 / 2}-0 f_{5 / 2}\right]$, which have the same pseudo angular momentum $\tilde{l}$ and the same $\widetilde{n}$, are, in the new notation, $\left[\widetilde{0} \widetilde{p}_{1 / 2}-\widetilde{0} \widetilde{p}_{3 / 2}\right]$ and $\left[\widetilde{0} \widetilde{d}_{3 / 2}-\widetilde{0}_{5 / 2}\right]$, respectively. Therefore, the harmonic oscillator with $\Sigma=U=0$ and $\Delta=\frac{1}{2} m \omega_{1}^{2} r^{2}$ provides an example of exact pseudospin symmetry. The fact that for this case we do not have a nonrelativistic limit, already shows the relativistic nature of the pseudospin symmetry. Figure 13(a) shows the $(2 \widetilde{n}+\widetilde{l})$ degeneracy, which means that not only states with same $\tilde{n}, \tilde{l}$ are degenerate (pseudospin partners) but also, for example, $(\tilde{n}-1, \tilde{l}+2)$ or $(\tilde{n}+1, \tilde{l}-2)$ have the same energy.

The singlet states $(\widetilde{l}=0)$ are the states $n p_{1 / 2}$ with $l=1$ inside the small dashed squares in Fig. 3. It is interesting to point out that the ground state level for this case is the state $0 p_{1 / 2}$ with $l=1$. This is an unusual behavior coming from the relativistic nature of this particular case.

\section{THE PSEUDOSPIN SYMMETRY AND THE INTRUDER LEVELS}

The concept of pseudospin symmetry appeared in nuclear physics more than 20 years ago $[19,20]$. It was introduced to account for the observation, in heavy nuclei, of the quasidegeneracy of orbitals with quantum numbers (for fixed $n$ and l)

$$
\left(n, l, j=l+\frac{1}{2}\right) \quad \text { and } \quad\left(n-1, l+2, j=l+\frac{3}{2}\right) .
$$

Such pairs of single particle states are known as pseudospin partners. 
The doublet structure in the spectrum is better expressed in terms of the pseudo-orbital angular moment and pseudospin quantum number $\tilde{l}=l+1$ and $\widetilde{s}=s=1 / 2$, respectively. The former, as noted by Ginocchio [12], is just the orbital angular momentum of the lower component of the Dirac spinor, introduced in Sec. II. The pseudospin partners have the same $\tilde{l}$. For example, for the partners $\left[n s_{1 / 2},(n-1) d_{3 / 2}\right]$, $\tilde{l}=1$, and for $\left[n p_{3 / 2},(n-1) f_{5 / 2}\right]$ one has $\tilde{l}=2$. Yet, the total angular momentum quantum number is still given by $j$ $=\widetilde{l} \pm \widetilde{s}$, where the aligned $(+)$ and unaligned $(-)$ cases are determined by the sign of $\widetilde{\kappa}$ [see Eq. (40)]. Pseudospin partners have $\widetilde{\kappa}$ with opposite signs (the states with $n$ and $n-1$ radial quantum numbers have, respectively, $\widetilde{\kappa}>0$ and $\widetilde{\kappa}<0$ ) and thus pseudospin and pseudo-orbital angular momentum have opposite alignments. The fact that $\tilde{l}$ is a good quantum number can then be related to the disappearance of the term with $\Sigma^{\prime}$ in Eq. (17), which can be interpreted as pseudospinorbit interaction term [21]. From that equation it is also clear that this happens only when $U=0$, that is, the tensor interaction breaks pseudospin symmetry.

The existence of pseudospin partners is connected to a SU(2) symmetry of the Dirac equation with only scalar and vector potentials when $\Delta=0$ or $\Sigma=0$, rather than to the particular shapes of these potentials. Back in 1975, Bell and Ruegg [15] obtained the pseudospin generators for that symmetry. In that pioneering work only the case $\Delta=0$ was studied with the possible application to meson spectra exhibiting a tiny spin-orbit splitting. More recently, Ginocchio considered the other case, $\Sigma=0$, to explain the quasidegeneracy of pseudospin doublets in nuclei. The generators of the symmetry for radial potentials were worked out in Ref. [16].

It is interesting to discuss how the pseudospin symmetry gets broken. The SU(2) generators of the pseudospin symmetry are given by $[8,15,16]$

$$
S_{i}=s_{i} \frac{1}{2}(1-\beta)+\frac{\boldsymbol{\alpha} \cdot \boldsymbol{p} s_{i} \boldsymbol{\alpha} \cdot \boldsymbol{p}}{p^{2}} \frac{1}{2}(1+\beta)=\left(\begin{array}{cc}
\widetilde{s}_{i} & 0 \\
0 & s_{i}
\end{array}\right),
$$

where

$$
\widetilde{s_{i}}=\frac{\boldsymbol{\sigma} \cdot \boldsymbol{p}}{p} s_{i} \frac{\boldsymbol{\sigma} \cdot \boldsymbol{p}}{p}=\frac{2 \boldsymbol{s} \cdot \boldsymbol{p}}{p^{2}} p_{i}-s_{i},
$$

and $s_{i}=\sigma_{i} / 2(i=1,2,3)$. The commutator of this operator with the Hamiltonian in Eq. (1) with $U=0$ is

$$
\left[H_{\mathrm{D}}, S_{i}\right]=\left(\begin{array}{cc}
{\left[\Sigma, \tilde{s}_{i}\right]} & 0 \\
0 & 0
\end{array}\right) .
$$

Thus, the pseudospin symmetry breaking can be related to the commutator $\left[\Sigma, \widetilde{s_{i}}\right][22]$. This commutator is zero when $\Sigma=0$, but for radial potentials it is enough that $\Sigma^{\prime}(r)=0$ [21]. The quasidegeneracy of some pseudospin partners can be seen in a Dirac Hamiltonian with scalar and vector potentials of Woods-Saxon type [13]. As explained before, one cannot set $\Sigma=0$ for those kind of potentials, so we cannot get the full degeneracy of all pseudospin partners. In fact, the nuclear pseudospin symmetry in nuclei has a dynamical character coming from a cancellation among different terms that contribute to the energy as discussed in several works [13,23-25].

We have seen in the previous sections that the relation between the nodes of radial functions depends on whether one has $\Delta=0$ or $\Sigma=0$ in Dirac Hamiltonian with harmonicoscillator potentials. Leviatan and Ginocchio have shown [14] that, in the limit of pseudospin symmetry, the lower radial functions of the pseudospin partners have the same number of nodes. Using this information, they were able to show [18] that, for scalar and vector potentials which go to zero at infinity, like Woods-Saxon potentials, the number of nodes is related by

$$
n_{f}=\left\{\begin{array}{l}
n_{g}, \quad \kappa<0 \\
n_{g}+1, \quad \kappa>0 .
\end{array}\right.
$$

This is the behavior we have found before for scalar and vector oscillator harmonic potentials with $\Delta=0$, Eq. (34), precisely the case for which we have the nonrelativistic harmonic-oscillator limit.

Let us now discuss the levels with $n_{g}=n_{f}=0$. From Eq. (67), we see that this can only happen when $j=l$ $+1 / 2(\kappa<0)$ but never with $j=l-1 / 2(\kappa>0)$. If we recall that the pseudospin doublets have the same $n_{f}$, in this case $n_{f}=0$, states such as $0 s_{1 / 2}, 0 p_{3 / 2}, 0 d_{5 / 2}$, etc., have no pseudospin partners. These states are known as intruder states. The $0 g_{9 / 2}$ state, for example, is an intruder and what would be its pseudospin partner - the state $0 i_{11 / 2}(\kappa=6)$ —does not exist because $n_{g}=n_{f}-1$ would be negative for $n_{f}=0$. This is what we observe in the nuclear spectra.

In the case of exact pseudospin symmetry $(\Sigma=0)$ with a harmonic-oscillator potential for $\Delta$, which we have discussed in Sec. III C, the structure of the nodes it is quite different. In accordance with Eq. (47),

$$
n_{f}=\left\{\begin{array}{l}
n_{g}-1, \quad \kappa<0 \\
n_{g}, \quad \kappa>0 .
\end{array}\right.
$$

Thus the intruder levels that have $n_{g}=n_{f}=0$ may only exist in the exact pseudospin limit when $j=l-1 / 2(\kappa>0)$, as we see from Eq. (68) and, in that sense, they are not the intruder levels seen in nuclei, where $j=l+1 / 2(\kappa<0)$.

In a recent paper the intruder levels have been discussed in the context of the relativistic harmonic oscillator [10] and it was suggested that they will have partners in the limit of the exact pseudospin symmetry $(\Sigma=0)$. However, in that paper, the pseudospin partners have been classified considering only the radial quantum number of the lower component (same $n_{f}$ ). In that classification, levels with the same $n_{f}=0$ may have partners according to Eq. (68). However, it seems that the relation between the number of nodes of the upper and lower components was considered to be given by Eq. (67) instead of the correct one given by Eq. (68). If we take into account that the nuclear intruder levels have $j=l$ $+1 / 2(\kappa<0)$ then, by Eq. (68), we see that states with negative $\kappa$ that have zero nodes in the lower component will have a node in the upper component, $n_{g}=1$. This disagrees with nuclear spectroscopy, since the intruder states should have $n_{g}=0$. This is shown in Fig. 13(a) where we show inside the 
solid square the state $1 g_{9 / 2}$ with $n=1$ and its partner $0 i_{11 / 2}$ that in the $(\tilde{n}, \widetilde{l})$ classification in Fig. 13(b) are the pseudospin doublet $\left[\tilde{0} \tilde{h}_{9 / 2}-\tilde{0} \tilde{h}_{11 / 2}\right]$.

We can summarize this discussion and conclude that even though the harmonic oscillator with $\Sigma=0$ has an exact pseudospin symmetry that could explain the almost degeneracy in $\tilde{l}$ observed in nuclei for the doublets with $j=l+1 / 2(\kappa<0)$, $j=l-1 / 2(\kappa>0)$, radial and orbital quantum numbers related as shown in Eq. (63), it cannot explain the origin of the intruder levels. Furthermore, the harmonic oscillator presents a much higher degeneracy in the energy levels besides the simple degeneracy $\tilde{l}$, as we have shown, which implies that the nuclear potential cannot be approximated by a harmonic oscillator and must be more complicated in order to remove this extra degeneracy. The asymptotic properties of the central mean-field potential also seem to play a crucial role in defining the node structure of the single particle levels.

\section{CONCLUSIONS}

We have presented a generalized harmonic oscillator for spin $1 / 2$ particles that includes not only the usual linear tensor potential obtained by a redefinition of the momenta, but also a vector $(V)$ and scalar potential $(S)$ that appear in the Dirac Hamiltonian in the specific combinations $1 / 2(1 \pm \beta) V$, in which $V$ is a harmonic-oscillator radial potential. A special attention was paid to the $1 / 2(1-\beta) V$ combination, i.e., when $S=-V(\Sigma=0)$, which has been related recently to the nuclear pseudospin symmetry.

We have derived the energy eigenvalue equations and shown explicitly the wave functions. The nonrelativistic limits for all cases have been discussed. The analytical expression for the upper and lower components of the Dirac spinor for $U=0$, when $\Sigma=0$ (or $\Delta=0$ ), has been found.

We have discussed the structure of the solutions of the eigenvalue equations and presented explicitly the positive energy solutions. We have concluded that only when the tensor potential is turned on we have the negative bound state solutions and in this case, when $\omega_{1}=0$, the two bound state solutions are symmetric. When the tensor potential is absent we have also shown that the special conditions between the scalar and vector potential $(S=V$ or $S=-V)$ needed to have an harmonic-oscillator potential with scalar and vector potentials exclude the negative bound state solutions from the spectra.

The structure of the radial nodes for the Dirac spinor has also been presented and compared to the case when the potentials vanish for large distances. We discussed in detail the case of the harmonic oscillator with $U=\Sigma=0$ for which pseu- dospin symmetry is exact, i.e., pseudospin doublets (states with same $\tilde{l}$ ) are degenerate. In this case we can have bound states due to the positive harmonic-oscillator potential $\Delta$. These states can be regarded as intrinsically relativistic, in the sense that the $\Delta$ potential has no analog in the nonrelativistic limit. In fact, they show a very peculiar structure of radial nodes in comparison to the nonrelativistic harmonicoscillator radial wave function: the states with $j=l+1 / 2$ have at least one node $(n \geqslant 1)$ in the upper component and can have zero nodes only in the lower one. As a consequence, the intruder levels on the nuclear spectra (isolated levels with $n=0$ and $j=l+1 / 2)$ do not exist in the harmonic oscillator in the limit of nuclear pseudospin symmetry $(\Sigma=0)$. In this limit the states with zero nodes in the upper component have $j=l-1 / 2$. Moreover, the relativistic harmonic oscillator in the case $U=\Sigma=0$ has a much higher energy degeneracy than the approximate pseudospin symmetry seen in the nuclear spectra (doublets with same $\widetilde{n}, \widetilde{l}$ ). This is not surprising since the meson exchange theory of nuclear forces produces nuclear potentials that vanish when $r \rightarrow \infty$, a completely different asymptotic behavior from the harmonic-oscillator potential discussed here. One possible way to break this extra degeneracy of the harmonic oscillator is to add a WoodsSaxon-like potential as it has been done in Ref. [10].

Another important conclusion from our work is that a Dirac equation with scalar and vector harmonic-oscillator potentials with the same sign and magnitude $(\Delta=0)$, instead of the linear tensor potential $U$, is the most natural way to introduce the harmonic oscillator in relativistic quantum mechanics, since one gets the correct nonrelativistic limit, so that the energy levels and the upper component of the wave functions are very similar to the nonrelativistic ones.

Finally, our analysis of the relativistic harmonic oscillator shows, in a very simple and analytical way, that the nuclear pseudospin symmetry $(\Sigma=0)$ does not have a nonrelativistic limit, in the sense that the eigenvalues are of second order in a $\omega / m$ expansion. So we conclude that the relativistic harmonic oscillator is an excellent example to show explicitly what Ginocchio had already emphasized in his pioneering work: the pseudospin symmetry in the Dirac Hamiltonian (when $\Sigma=0$ ) is indeed a symmetry of relativistic nature.

\section{ACKNOWLEDGMENTS}

We acknowledge financial support from CNPq, FAPESP, and FCT (POCTI) scientific program. R.L. and M.M. acknowledge, in particular, the CNPq support and A.S.C. was also supported by FAPESP. P.A. and M.F. were supported in part by FCT (Lisbon), Project No. POCTI/FIS/451/1994.
[1] D. Itó, K. Mori, and E. Carriere, Nuovo Cimento A 51, 1119 (1967); N. V. V. J. Swamy, Phys. Rev. 180, 1225 (1969); P. A. Cook, Lett. Nuovo Cimento 10, 419 (1971); E. F. Chaffin, J. Math. Phys. 14, 977 (1973); Y. M. Cho, Nuovo Cimento A 23,
550 (1974); H. Ui and G. Takeda, Prog. Theor. Phys. 72, 266 (1984); 72, 813 (1984); A. B. Balantekin, Ann. Phys. (N.Y.) 164, 277 (1985); J. N. Gnocchio, in Symmetries in Science II, edited by B. Gruber and R. Lenczewski (Plenum, New York, 
1986), p. 75; R. J. Hughes, V. A. Kostelecky, and M. M. Nieto, Phys. Rev. D 34, 1100 (1986).

[2] M. Moshinsky and A. Szczepaniak, J. Phys. A 22, L817 (1989).

[3] M. Moreno and A. Zentella, J. Phys. A 22, L821 (1989); J. Benitez, R. P. Martinez y Romero, H. N. Nuñez-Yépez, and A. L. Salas-Brito, Phys. Rev. Lett. 64, 1643 (1990); F. Domínguez-Adame and M. A. González, Europhys. Lett. 13, 193 (1990); M. Moreno, R. Martínez, and A. Zentella, Mod. Phys. Lett. A 5, 949 (1990); M. Moshinsky, G. Loyola, and C. Villegas, J. Math. Phys. 32, 373 (1991); C. Quesne, Int. J. Mod. Phys. A 6, 1567 (1991); O. Castaños, A. Frank, R. López, and L. F. Urrutia, Phys. Rev. D 43, 544 (1991).

[4] M. Moshinsky, G. Loyola, and A. Szczepaniak, The Two-Body Dirac Oscillator (World Scientific, Singapore, 1990); M. Moshinsky, G. Loyola, A. Szczepaniak, C. Villegas, and N. Aquino, in Proceedings of the International Workshop on Relativistic Aspects of Nuclear Physics, Rio de Janeiro, Brazil, 1990, edited by T. Kodama et al. (World Scientific, Singapore, 1990), pp. 271-303.

[5] C. Quesne and M. Moshinsky, J. Phys. A 23, 2263 (1990); O. L. de Lange, ibid. 24, 667 (1991); R. P. Martinez y Romero, H. N. Nuñez-Yépez, and A. L. Salas-Brito, Eur. J. Phys. 16, 135 (1995); M. Moshinsky, C. Quesne, and Y. F. Smirnov, J. Phys. A 28, 6447 (1995).

[6] V. M. Villalba, Phys. Rev. A 49, 586 (1994); P. Rozmej and R. Arvieu, J. Phys. A 32, 5367 (1999); V. M. Villalba and A. A. R. Maggiolo, Eur. Phys. J. B 22, 31 (2001).

[7] Y. Nogami and F. M. Toyama, Can. J. Phys. 74, 114 (1996); F. Toyama, Y. Nogami, and F. A. B. Coutinho, J. Phys. A 30, 2585 (1997); F. M. Toyama and Y. Nogami, Phys. Rev. A 59, 1056 (1999); P. Rozmej and R. Arvieu, J. Phys. A 32, 5367 (1999); V. M. Villalba and A. A. R. Maggiolo, Eur. Phys. J. B 22, 31 (2001); M. H. Pacheco, R. Landim, and C. A. S. Almeida, Phys. Lett. A 311, 93 (2003).

[8] B. Smith and L. J. Tassie, Ann. Phys. (N.Y.) 65, 352 (1971); F. Ravndal, Phys. Lett. 113B, 57 (1982); R. Tegen, Ann. Phys. (N.Y.) 197, 439 (1990); M. Centelles, X. Viñas, M. Barranco, and P. Schuck, Nucl. Phys. A519, 73c (1990); Q. W.-Chao,
Chin. Phys. 11, 757 (2002).

[9] V. I. Kukulin, G. Loyola, and M. Moshinsky, Phys. Lett. A 158, 19 (1991).

[10] T.-S. Chen, H.-F. Lü, J. Meng, S.-Q. Zhang, and S.-G. Zhou, Chin. Phys. Lett. 20, 358 (2003).

[11] J. N. Ginocchio, (private communication), to appear in Phys. Rev. C.

[12] J. N. Ginocchio, Phys. Rev. Lett. 78, 436 (1997); Phys. Rep. 315, 231 (1999).

[13] P. Alberto, M. Fiolhais, M. Malheiro, A. Delfino, and M. Chiapparini, Phys. Rev. Lett. 86, 5015 (2001); Phys. Rev. C 65, 034307 (2002).

[14] J. N. Ginocchio and A. Leviatan, Phys. Rev. Lett. 87, 072502 (2001).

[15] J. S. Bell and H. Ruegg, Nucl. Phys. B98, 151 (1975).

[16] J. N. Ginocchio and A. Leviatan, Phys. Lett. B 245, 1 (1998).

[17] Handbook of Mathematical Functions, edited by M. Abramowitz and I. Stegun (Dover Publications, New York, 1972).

[18] A. Leviatan and J. N. Ginocchio, Phys. Lett. B 518, 214 (2001).

[19] K. T. Hecht and A. Adler, Nucl. Phys. A137, 129 (1969).

[20] A. Arima, M. Harvey, and K. Shimizu, Phys. Lett. B30, 517 (1969).

[21] J. Meng, K. Sugawara-Tanabe, S. Yamaji, P. Ring, and A. Arima, Phys. Rev. C 58, R628 (1998).

[22] J. Dudek, P. Casoli, N. Schunck, D. Valet, and Z. Łojewski, Acta Phys. Pol. B 30, 771 (1999).

[23] R. Lisboa, M. Malheiro, and P. Alberto, Phys. Rev. C 67, 054305 (2003).

[24] S. Marcos, L. N. Savushkin, M. López-Quelle, and P. Ring, Phys. Rev. C 62, 054309 (2000).

[25] S. Marcos, M. López-Quelle, R. Niembro, L. N. Savushkin, and P. Bernardos, Phys. Lett. B 513, 30 (2001).

[26] M. G. Salvadori, Numerical Methods in Engineering (PrenticeHall, New York, 1952).

[27] I. S. Berezin and N. P. Zhidkov, Computing Methods (Pergamon, Oxford, 1965), Vol. II. 